\begin{tabular}{|l|l|l||}
\hline \multicolumn{2}{|c|}{ PublisherInfo } \\
\hline \hline PublisherName & $:$ & BioMed Central \\
\hline \hline PublisherLocation & $:$ & London \\
\hline \hline PublisherImprintName & $:$ & BioMed Central \\
\hline \hline
\end{tabular}

\title{
The role of VEGF in bone formation
}

\begin{tabular}{|l|l|l||}
\hline \multicolumn{2}{|c||}{ ArticleInfo } \\
\hline \hline ArticleID & $:$ & 12 \\
\hline \hline ArticleDOI & $:$ & $10.1186 /$ ar-2000-66786 \\
\hline \hline ArticleCitationID & $:$ & 66786 \\
\hline \hline ArticleSequenceNumber & $:$ & 8 \\
\hline \hline ArticleCategory & $:$ & Paper Report \\
\hline \hline ArticleFirstPage & $:$ & 1 \\
\hline \hline ArticleLastPage & $:$ & 3 \\
\hline \hline & & RegistrationDate : 2000-2-22 \\
\hline ArticleHistory & $:$ & OnlineDate \\
\hline \hline ArticleCopyright & $:$ & Current Science Ltd2000-2-22 \\
\hline \hline ArticleGrants & $:$ & \\
\hline \hline ArticleContext & $:$ & 130752211 \\
\hline \hline
\end{tabular}


Aff1 Kennedy Institute of Rheumatology, USA

\section{Keywords}

Development, endochondral bone formation, neovascularisation, VEGF

\section{Context}

During the process of endochondral ossification the cartilaginous bone rudiment becomes vascularised, beginning in the diaphysis and continuing in the growth plate. Different stages of chondrocyte differentiation are apparent in the growth plate: resting; proliferating and hypertrophic chondrocytes; a zone of mineralisation; and a zone of blood vessel invasion and cartilage invasion, where ossification occurs. New blood vessel formation plays a major role in endochondral ossification. This process is controlled by a fine balance of stimuli and inhibitors, of which VEGF is perhaps the best characterised pro-angiogenic factor. A disruption of this angiogenic balance may promote conditions associated with pathological neovascularisation, including RA. Vascularisation of cartilage is also a feature of OA. To establish the function of VEGF-stimulated angiogenesis in endochondral bone formation.

\section{Significant findings}

Cartilage hypertrophy precedes vascularisation of the bone rudiment. Using immunohistochemistry, VEGF was found to be present in mammalian and avian long bones, in fully mature chondrocytes, sporadically in pre-hypertrophic chondrocytes, but not in proliferating and quiescent chondrocytes. Using the culture system of avian chondrocyte differentiation, VEGF was found to be released by chondrocytes under conditions leading to the formation of engineered hypertrophic cartilage. VEGF was expressed around the lacunae and in the interposed extracellular matrix. Culture supernatants from hypertrophic chondrocytes markedly stimulated endothelial migration and invasion. This effect was blocked by antibodies to VEGF, although the inhibition was only partial, suggesting that other proangiogenic factors also play a role. Similarly, antibodies against the VEGF receptor thought to be responsible for the pro-angiogenic effect of VEGF, Flk-1 (VEGF receptor type 2) also partially reduced the chemotactic migration and invasion of HMEC-1 induced by chondrocyte-released VEGF. Indeed, Flk-1 colocalised with VEGF both in vivo in sections of mouse and chick tibiae and in the hypertrophic chondrocyte region, but not in the zone of quiescent chondrocytes, and also in the in vitro engineered cartilage. The concerted expression of VEGF and Flk-1 in hypertrophic cartilage suggests that VEGF 
may act as an autocrine signal for cells of the chondrogenic lineage. Western blot analysis using antibodies against Flk-1 and phosphotyrosine confirmed that Flk-1 is expressed in hypertrophic chondrocytes and is phosphorylated. Moreover, this phosphorylation is independent of the presence of VEGF.

\section{Comments}

To date, vascular endothelial growth factor (VEGF) has been thought to be primarily involved in promoting angiogenesis, both as part of normal bone development and during angiogenesis-dependent pathologies such as rheumatoid arthritis (RA) and osteoarthritis (OA). This paper confirms that VEGF is expressed in bones prior to the onset of vascular invasion, and additionally describes a possible new role for VEGF in chondrocyte activation. These data are relevant in terms of increasing our understanding of the role of angiogenesis in bone development and in the pathogenesis of arthritis. In particular, subchondral vascular responses, and especially cartilage neovascularisation, may contribute to the features of OA. Anti-angiogenic therapies are in clinical trials for the treatment of malignancies, and have been put forward as an adjunct to conventional therapies for RA. This publication suggests that we have a lot to learn about the role of the angiogenic cytokine VEGF in terms of its effects on joint development and the pathology of arthritis.

\section{Methods}

Immunohistochemical analyses for VEGF and the VEGF receptor Flk-1 were performed on sections of chick and mouse embryo limbs of different ages. To measure VEGF synthesis by hypertrophic chondrocytes, a culture system for avian chondrocyte differentiation was used. Chondrocytes were prepared from chick embryo tibiae. The chondrocyte phenotype was resumed and differentiation to hypertrophy continued on transferring cells into suspension culture in agarose-coated dishes. Suspension culture of cells in the presence of ascorbic acid led to the formation of in vitro engineered cartilage. The chemotactic and chemoinvasive properties of chondrocyte conditioned medium were assayed against a human microvascular endothelial cell line (HMEC)-1.

\section{References}

1. Carlevaro MF, Cermelli S, Cancedda R, Descalzi Cancedda F: Vascular endothelial growth factor (VEGF) in cartilage neovascularisation and chondrocyte differentiation: auto-paracrine role during endochondral bone formation. J Cell Sci. 2000, 113: 59-69.

This PDF file was created after publication. 